\title{
Proyecciones
}

Vol. $12 \mathrm{~N}^{0} 2$, pp.149-153 Deceruber 1993

Universidad Católica del Norte

Antofagasta - Chile

\section{ON THE CODOMINATION NUMBER OF A GRAPH}

\author{
FRANK HARARY \\ New Mexico State University, Las Cruces, USA. \\ TERESA W. HAYNES \\ East Tennessee State University, Johnson City, USA. \\ MARTIN LEWINTER \\ State University of New York at Purchase, Purchase, USA.
}

\begin{abstract}
Given a graph $G=(V, E)$, set $S \subset V$ is a dominating set if each node of $V-S$ is adjacent to at least one node in $S$. The domination number of $G$ is the smallest size of a dominating $s+$ and the codomination number is the domination number of its complement. We determine the codomination number of a graph having dameter at least three. Further we explore the effects of this result on the open problem of characterizing graphs having equal domination and codomination numbers.
\end{abstract}




\section{Introduction.}

In general, we follow the notation and terminology of [8]. Each node of a graph $G=(V, E)$ dominates every node in its closed neighborhood. A sei $S \subset V$ is a dominating set if each node in $V$ is dominated by some node of $S$. The domination number $d=d(G)$ is the smallest size of a dominating set. The domination number $\bar{d}=d(\bar{G})$ of its complement is called the codomination number of $G$. Domination in both a graph and its complement has been the topic of several recent papers $[2,3,5,6,7,15,16]$. These authors were concerned with finding a smallest set $S \subset V$ which dominates both $G$ and $\bar{G}$, called a "factor dominating set" in [5] and a "global dominating set" in [16]. Others [9, 12, 13] found NordhausGaddum type inequalities involving domination and codomination numbers. For a recent bibliography on domination see [11]. The investigation of graphs $G$ for which an arbitrary invariant has the same value in $G$ and $\bar{G}$ is surveyed in [1].

The diameter, denoted $\operatorname{diam}(G)$, is the maximum distance between any two nodes of $G$. We establish a relationship between the diameter of a graph and its codomination number and consider the ramification of this results on characterizing graphs for which $d=\bar{d}$.

\section{Codomination Number.}

A bound on the diameter of $\bar{G}$ for the case when $\operatorname{diam}(G) \geq 3$ is given in [10] as a lemma in proving that every nontrivial self-complementary graph has dianeter 2 or 3 .

Theorem A. [10] If $\operatorname{diam}(G) \geq 3$, then $\operatorname{diam}(\bar{G}) \leq 3$.

Furthermore, it is shown in [14] that if "regular" is added to the liypotheses, then the diameter of the complement is at most two. Brigham, Chinn, and Dutton [4] obtained an interesting relationship between the diameter and codomination number of a graph.

Theorem B. [4] If $\bar{d} \leq 3$, then $\operatorname{diam}(G) \leq 2$.

If $G$ is not connected, let $\operatorname{diam}(G)=\infty$. We now determine the codomination number of any graph having diameter at least three.

Theorem 1. If $G$ has no isolated nodes and $\operatorname{diam}(G) \geq 3$, then $\bar{d}=2$.

Proof. Let $x$ and $y$ be nodes of $G$ such that $d(x, y)=\operatorname{diam}(G) \geq 3$. Obviously, $x$ and $y$ form a dominating set for the complement of $G$. For any node $z$ which is adjacent to neither $x$ nor $y$ in $\bar{G}$ is adjacent to both nodes in $G$, contrary to the hypothesis that $d(x, y) \geq 3$. It follows that the $\bar{d} \leq 2$. If $\bar{d}=1$, then $G$ would have an isolated node, again contrary to the hypothesis. 
Although we have not characterized graplıs which have equal domination and codomination numbers, our next result provides insigltt about their structure.

Corollary 1.1. Let $G$ be a nontrivial connected graph having $d=\bar{d}$. Then either $d=\bar{d}=2$ or $\operatorname{diam}(G)=2$.

Proof. Let $G$ be a nontrivial graph with $d=\bar{d}$. If $\operatorname{diam}(G) \geq 3$, then $d=\bar{d}=2$ by Theorem 1. If $\operatorname{diam}(G)=1$, then $\bar{G}$ is discomnected and $d=1 \neq \bar{d}$. In all other cases $\operatorname{diam}(G)=2$.

Corollary 1.2. Cycles $C_{n}$ and paths $P_{n}$ have $d=\bar{d}$ if and only if $n=4,5,6$.

Obviously, self-complementary graphs have $d=\bar{d}$ and as mentioned above it follows directly from Theorem A that every nontrivial self-complementary graph has diameter 2 or 3 .

Corollary 1.3. If $G$ is a nontrivial self-complementary graph, then $\operatorname{diam}(G)=$ $\operatorname{diam}(\bar{G})=2$ or $\operatorname{diam}(G)=\operatorname{diam}(\bar{G})=3$ and $d=\bar{d}=2$.

\section{Examples.}

(1) The path $P_{4}$ is a self-complementary graph having $d=\bar{d}=2$ and diam $=3$.

(2) The composition of $C_{5}$ with itself, written $G=C_{5}\left[C_{5}\right]$, is a self-complementary graph laving $d=\bar{d}=3$ and diam $=2$.

\section{REFERENCES}

[1] Akiyama, J.; Harary F.: A graph and its complement with specified properties VII: A survey. G. Chartrand, ed., The Theory and Applications of Graphs. Wiley, New York, (1981), 1-12.

[2] Brigham, R.C.; Carrington, J.R.: Factor domination. Congr. Numer., 83 (1991), 201-211.

[3] Brigham, R.C.; Carrington, J.R.: Global domination of simple factors. Congr. Numer., 88 (1992), 161-167.

[4] Brigham, R.C.; Chinn, P.Z.; Dutton, R.D.: Vertex domination-critical graphs. Networks 18 (1988), 173-179.

[5] Brigham, R.C.; Dutton, R.D.: Factor domination in graphs. Discrete Math., 86 (1990), 127-136. 
[6] Dunbar, J.: Laskar, R.; Monroe, T.: Global irredundant sets in graphs. Congr. Numer., 85 (1991), 65-72.

[7] Dunbar, J.: Laskar, R.; Monroe, T.: Some global parameters of graphs. Congr. Numer., 89 (1992), 187-191.

[8] Harary, F.: Graph Theory. Addison-Wesley, Reading, 1969.

[9] Harary, F.; Haynes T.W.: Nordhaus-Gaddum inequalities on graplı dominatioll. Networks, to appear.

[10] Harary, F.; Robinson, R.W.: The diameter of a graph and its complement. Amer. Math. Monthly, 92 (1985), 211-212.

[11] Hedetniemi, S.T.; Laskar, R.: Bibliography on domination in graphs and some basic definitions of domination parametes. Discrete Math., 86 (1990), 257-277.

[12] Jaeger, F,; Payan, C.: Relations du type Nordhaus-Gaddum pour le nombre d'absorption d'un graph simple. C. R. Acad. Sci. Paris, A 274 (1972), 728730 .

[13] Laskar, R.; Peters, K.: Vertex and edge domination parameters in graphs. Congr. Numer., 48 (1985), 291-305.

[14] Lewinter, M.: The diameter of the complement of a regular grapl. Congr. Numer., 64 (1988) 157-158.

[15] Rall, D.: Dominating a graph and its compement. Congr. Numer., 64 (1988) 157-158.

[16] Sampathkumar, E.: The global domination number of a graph. J. Math. Phy. Sci., 23 (1989) 377-385.

Received: September 23, 1993.

Frank Harary

Department of Computer Science

New Mexico State University

Las Cruces, NM 88003-0001, USA.

Teresa W. Haynes

Department of Computer Science

East Tennessee State University

Johnson City, TN 37614-0002, USA. 
Martin Lewinter

Department of Mathematics

State University of New York at Purcliase.

Purchase, NY 10577, USA. 